\title{
Improved immunoturbidimetric method for rheumatoid factor testing
}

\author{
M Nykänen, T Palosuo, K Aho, T Sahi, R von Essen
}

\begin{abstract}
The performance of two immunoturbidimetric modifications for rheumatoid factor (RF) testing, which differ with respect to the means of complement inactivation (heat treatment and inactivation with polyvinyl sulphonate), were compared in serum samples from 87 patients with rheumatoid arthritis (RA) and from 403 healthy subjects. IgM-rheumatoid factor titres were also measured with an enzyme linked immunosorbent assay (ELISA). Both immunoturbidimetric tests gave positive reactions (rheumatoid factor $\geqslant 20$ IU/ml) in 74 out of the $87(85 \%)$ RA sera. In cases with high RF concentrations the results after chemical inactivation tended to be slightly higher compared with heat inactivation. In healthy subjects rheumatoid factor was detected in 19/403 (4.7\%) sera using heat inactivation and in 22/403 $(5 \cdot 5 \%)$ sera with chemical inactivation of complement. Interrun coefficient of variation in the chemical inactivation assay was 4.4\%; with the heat inactivation method it was $8 \cdot 1 \%$. In the ELISA, a marginally better correlation was noted in the results obtained using chemical inactivation.

Inactivation of complement by means of polyvinyl sulphonate offers the advantage of easier test performance and better reproducibility, and the results may reflect more accurately true rheumatoid factor concentrations.
\end{abstract}

\section{(F Clin Pathol 1993;46:1065-1066)}

Several methods for rheumatoid factor (RF) measurement, including nephelometric and turbidimetric assays, have the disadvantage of requiring prior heat inactivation of serum to prevent the binding of complement component $\mathrm{Clq}$ to the test antigen. It has been reported that heat inactivation can be avoided by using a chemical inhibitor of $\mathrm{Clq}$, polyvinyl sulphonate. ${ }^{1}$ Some serum samples negative after heat inactivation were weakly positive by the new method, ${ }^{2}$ but no details for critical assessment of the relevance of this observation were given. As the above may influence the sensitivity and specificity of the assay we decided to study the question further.

\section{Methods}

Sera from 87 patients with rheumatoid arthritis (13 men and 74 women) from the Rheumatism Foundation Hospital, Heinola, Finland, were included in the study. Median age of the patients was 60 (range 24-81) years and 67 of them had had rheumatoid arthritis for at least 20 years.
Sera from 403 selectively healthy people (331 men and 72 women) working at the Lapland Frontier Guard District comprised a Finnish population sample. Their mean age was 42 (range 19-60) years. All sera were stored as aliquots at $-70^{\circ} \mathrm{C}$ and had been thawed only once before testing.

\section{ASSAYS FOR RHEUMATOID FACTOR}

For immunoturbidimetric measurement of rheumatoid factor, a Kone Specific automated clinical chemistry analyser (Kone Instruments, Espoo, Finland) was used. Immunoturbidimetry using heat inactivation of the sera to destroy complement was performed according to the method of Melamies et $a l^{3}$ using a commercial kit for rheumatoid factor determination (Orion Diagnostica, Espoo, Finland). Serum specimens were heated at $56( \pm)^{\circ} \mathrm{C}$ to inactivate complement, aggregated human IgG was used as the antigen for rheumatoid factor, and the reference serum was calibrated against the WHO rheumatoid factor standard. The results were expressed as $\mathrm{IU} / \mathrm{ml}$.

Immunoturbidimetry using chemical inactivation of complement was performed essentially as described by Borque et al, ${ }^{1}$ except that a different instrument (endpoint turbidimeter instead of a centrifugal analyser) was used. The chemical inactivator of complement was sodium salt of poly(vinyl sulphonic acid), purchased from Aldrich Chemical Company, Milwaukee, Wisconsin, USA, in borate buffer (32 $\mathrm{g} / \mathrm{l}$ ). Reproducibilities of both of the immunoturbidimetric assay modifications were compared by testing one moderate level rheumatoid factor control serum $(75 \mathrm{IU} / \mathrm{ml})$ on 21 successive runs. Interrun coefficient of variation $(\mathrm{CV})$ in the chemical inactivation assay was $4 \cdot 4 \%$, in the heat-inactivation method it was $8 \cdot 1 \%$.

\section{QUANTIFICATION OF IGM-RF BY ELISA}

The method described in detail by Tuomi ${ }^{4}$ was used with minor modifications. Briefly, polystyrene microtitre plates (Immunoplate I, Nunc, Roskilde, Denmark) were coated with purified Fc fragments of human IgG $(2.5$ $\mu \mathrm{g} / \mathrm{ml}$; Jackson Immunoresearch Laboratories, West Grove, Philadelphia) and IgM-RF bound from the test sera was detected by alkaline phosphatase conjugated $F(a b ')_{2}$-fragment of Fc $\mu$-specific anti-human IgM (Jackson Immunoresearch). The colour formed in reaction with $p$-nitrophenyl-phosphate was read in a Titertek Multiskan spectrophotometer (Eflab, Helsinki, Finland). Rheumatoid factor standard serum diluted to contain $15 \cdot 25$, $30 \cdot 5,61$ and $122 \mathrm{IU} / \mathrm{ml}$ was included in each plate and the optical density values for each serum were converted to $\mathrm{IU} / \mathrm{ml}$. All determinations were done in duplicate. Interrun $(n=$ 38) $\mathrm{CV}$ at low to moderate rheumatoid factor 
Comparison of assay systems for detection of rheumatoid factor (Spearman's rank correlations)

\begin{tabular}{ll}
\hline & Correlation \\
\hline Rheumatoid sera $(n=87)$ & \\
Turbidimetry heat inactivation/turbidimetry chemical inactivation & 0.99 \\
Turbidimetry heat inactivation//gM-RF ELISA & 0.94 \\
Turbidimetry chemical inactivation/IgM-RF ELISA & 0.95 \\
Control sera $(n=403)$ & 0.38 \\
Turbidimetry heat inactivation/turbidimetry chemical inactivation & $0 \cdot 19$ \\
Turbidimetry heat inactivation/IgM-RF ELISA & $0 \cdot 22$ \\
Turbidimetry chemical inactivation/IgM-RF ELISA & \\
\hline
\end{tabular}

concentration $(40 \mathrm{IU} / \mathrm{ml})$ was $15 \cdot 3 \%$ and at a high rheumatoid factor concentration (400 $\mathrm{IU} / \mathrm{ml}) \mathbf{7 \cdot 9 \%}$.

\section{Results}

Positive rheumatoid factor test results $(\geqslant 20$ $\mathrm{IU} / \mathrm{ml}$ ) were recorded in $74 / 87(85 \%)$ rheumatoid arthritis sera by both immunoturbidimetric assays. Among the selectively healthy subjects immunoturbidimetry using heat inactivation of complement gave positive results in $19 / 403(4 \cdot 7 \%)$ sera while chemical inactivation demonstrated rheumatoid factor in $22 / 403(5.5 \%)$ sera. IgM-RF ELISA gave positive results in $86 \%$ of the rheumatoid arthritis sera and in $5.5 \%$ of the control sera.

There was in rheumatoid arthritis sera a good correlation between the results by the two immunoturbidimetric assays (table and figure). In sera with rheumatoid factor concentrations above $100 \mathrm{IU} / \mathrm{ml}$ the results when using chemical inactivation tended to be slightly higher. No corresponding phenomenon was seen in sera with rheumatoid factor concentrations below $100 \mathrm{IU} / \mathrm{ml}$. In all control sera the rheumatoid factor concentrations, as measured by the two immunoturbidimetric assays, were below $100 \mathrm{IU} / \mathrm{ml}$, and

Measurement of rheumatoid factor by immunoturbidimetry in rheumatoid arthritis ( $A$ ) and control $(B)$ sera. Correlation between chemical inactivation and heat-inactivation of complement. Rheumatoid factor results

$\geqslant 10 \mathrm{IU} / \mathrm{ml}$ are... depicted.
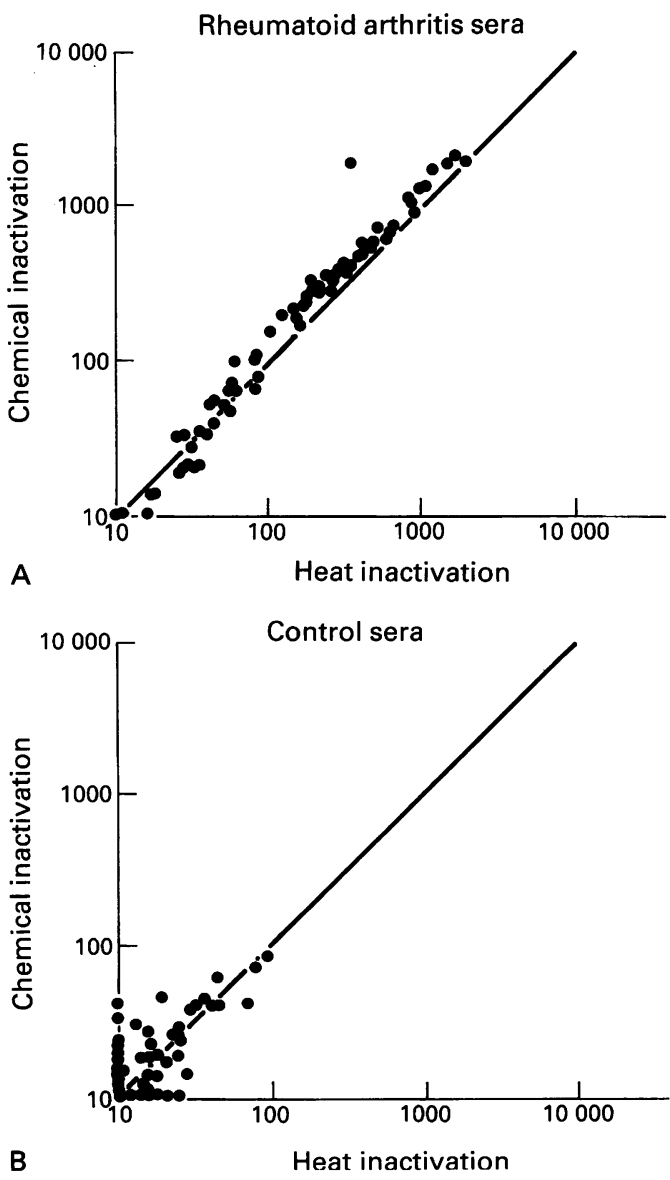

both higher and lower test results were recorded when using chemical inactivation (figure). There was only a modest correlation in the control sera between the two assays (table).

The correlations between the results by the two immunoturbidimetric assays were better than those between IgM-RF ELISA and each of the immunoturbidimetric assays (table). Both in rheumatoid arthritis sera and in control sera a marginally better correlation was noted between IgM-RF ELISA and immunoturbidimetric assay using chemical inactivation of C1q compared with the assay using heat inactivation.

\section{Discussion}

Our observations agree with earlier findings showing a good correlation between the results of the immunoturbidimetric rheumatoid factor assays using heat inactivation and chemical inactivation of C1q. ${ }^{1}$ Yet in sera with high rheumatoid factor concentrations the results by the latter modification tended to be slightly higher. This may be due to binding of rheumatoid factor to autologous aggregated IgG induced by heat treatment or, perhaps more likely, to a direct inactivation of some rheumatoid factor moieties due to this procedure. For some reason, a corresponding trend was not observed in sera with lower rheumatoid factor concentrations. There was no appreciable difference between the two modifications in the frequency of positive reactions either in rheumatoid arthritis sera or in control sera.

The correlation between the two immunoturbidimetric modifications was poor in sera with rheumatoid factor concentrations below $20 \mathrm{IU} / \mathrm{ml}$ (figure). We have already shown that correlations in non-rheumatoid sera between immunoturbidimetric assay, latex slide test, and IgM-RF ELISA were also poor when rheumatoid factor concentrations were below $20 \mathrm{IU} / \mathrm{ml} .{ }^{5}$ Marginally better correlation was observed in the present series between IgM-RF ELISA and immunoturbidimetry after chemical inactivation of $\mathrm{Clq}$ than after heat-inactivation both in rheumatoid arthritis sera and in control sera. Thus addition of polyvinyl sulphonate to the reagent buffer offers the advantage of easier performance of immunoturbidimetric testing. Results obtained by this modification may reflect more accurately true rheumatoid concentrations, and sensitivity and specificity of the assay remain essentially the same.

1 Borque L, Rus A, Ruiz R. Automated turbidimetry of rheumatoid factor without heat inactivation of serum Eur f Clin Chem Clin Biochem 1991;29:521-7.

2 Borque L, Ruiz R, Rus A. Healthy individuals seropositive for rheumatoid factor. Clin Chem 1992;38:1508.

3 Melamies LM, Ruutsalo HM, Nissilä M. Evaluation of a quantitative immunoturbidimetric assay for rheumatoid factors. Clin Chem 1986;32:1890-4.

4 Tuomi $T$. Which antigen to use in the detection of rheumatoid factors? Comparison of patients with rheumatoid factors? Comparison of patients with rheumatoid arthritis and subjects with "false positive" rheumatoid

5 Ailus K, Melamies L, Tuomi T, Palosuo T, Aho K Measuring rheumatoid factor in nonrheumatoid subjects: Immunoturbidimetric assay, latex slide test, and jects: Immunoturbidimetric assay, latex slide test, and enzyme-linked immun 